\title{
LOS VIAJES FRACASADOS DE VESPUCCI A CATTIGARA, TAPROBANA Y MALACA.
}

\author{
Por ENRIQUE DE GANDIA \\ da Academia Nacional de la Historia (Buenos Aires).
}

Los estudios sobre los viajes de Américo Vespucci, en español Vespucio, son muchos y todos ellos se contradicen y enturbian en sus análisis e interpretaciones. La bibliografia, en verdad numerosa, es bien conocida por los especialistas. Todo nuevo trabajo hace suponer que nada más podrá agregarse a las discusiones que comenzaron con Las Casas y no se han detenido en el presente. La autenticidad de sus escritos, impresos en su tiempo unos y descubiertos más tarde otros, encuentran aún negadores. Nosotros hemos intentado demostrar su autenticidad comparando las menciones que Vespucci hace de unas cartas en otras de sus cartas. Es decir: creemos que las cartas de Vespucci bastan, por si solas, con sus continuas referencias, para probar que han tenido un único autor (1). En otros escritos hemos pretendido llevar a Vespucci hasta el paralelo $52^{\circ}$ de Latitud Sud sin apartase de la costa, hasta que una tormenta, como él mismo refiere, lo arrastró a una tierra que sólo puede ser la de las islas. Malvinas (2). En estas páginas buscamos nuevas aclaraciones. En primer término sabemos por sus Quatuor Americi Vesputii navigationes o la Lettera di Amerigo Vespucci delle isole nuovamente trovate in quattro suoi viaggi (3) que escribió a Pier Soderini, gonfalonero perpetuo de Flo-

(1). - ENRIGUE DE GANDIA, La autenticidad del epistolario vespuciano, en ACADEMIA NACIONAL DE LA HISTORIA, Investigaciones $y$ Ensayos, Buenos Aires, 1967, II, 9-36.

(2). - ENRIQUE DE GANDIA, Claudio Alejandro Ptolomeo, Colón y la exploración de la India Americana, en ACADEMIA NACIONAL DE LA HISTORIA, Investigaciones y Ensayos, Buenos Aires, 1972, XIII, 35-87.

(3). - Seguimos, en todos los escritos de Vespucci, los textos en italiano y su traducción al español que publicó ROBERTO LEVILLIER en AMERICO VESPUCIO, El Mundo Nuevo, cartas relativas a sus viajes y descubrimientos, Editorial Nova, Buenos Aires, 1951. 
rencia, fechada en Lisboa el 4 de septiembre de 1504, que partió de Cádiz el 10 de mayo de 1497 por orden del rey don Fernando de Castilla, en cuatro naves,

"a descubrir nuevas tierras hacia el occidente" (4).

El propósito de Vespucci fue, por tanto, el de navegar en busca de nuevas tierras. Lo repite al comienzo de su Lettera:

"Benvenuto Benvenuti, nuestro florentino... me rogó diese parte a Vuestra Magnificencia de las cosas vistas por mi en diversas regiones del mundo, en cuatro viajes que hice para descubrir nuevas tierras: dos por orden del rey de Castilla, don Fernando VI (sic por V), por el gran golfo del mar Océano hacia el Occidente, y los otros dos por mandato del rey don Manuel de Portugal hacia el austro" (5).

Debemos empezar por destacar dos hechos: los viajes que Vespucci hizo por orden del rey de España eran "hacia el Occidente" y los que hizo por orden del rey de Portugal eran "hacia el austro": dos direcciones muy diferentes que es preciso tener en cuenta.

El itinerario de este primer viaje, como todos los suyos, ha sido discutido. No entramos en estas polémicas. Lo seguro es que avanzó en el mar Caribe y tocó una tierra, al Noroeste de la península de Yucatán, que llamó Lariab.

"Vinieron a vernos muchas gentes, y se maravillaban de nuestra figura y de nuestra blancura, y nos preguntaron de dónde veníamos, y les dábamos a entender que veníamos del cielo y que andábamos viendo el mundo, y lo creían. En esta tierra pusimos pila bautismal e infinita gente se bautizó; y en su lengua nos llamaban carabi, que quiere decir varones de gran sabiduría. Partimos de este puerto; la provincia se llama Lariab; y navegamos a lo largo de la costa siempre a vista de la tierra, tanto que recorrimos de ella ochocientas setenta leguas" (6).

Vespucci dice que "la provincia se llama Lariab". Había llegado, por tanto, a la provincia de Lariab. Rogamos que se recuerde este nombre.

(4). - AMERICO VESPUCIO, El Mundo Nuevo cit. 203.

(5). - AMERICO VESPUCIO, El Mundo Nuevo cit. 201.

(6). - AMERICO VESPUCIO, El Mundo Nuevo cit. 229. 
Algunos historiadores han dicho que Lariab es una deformación de otro nombre mencionado por Vespucci: Paria. Otros estudiosos, como el gran Varnhagen, lo han identificado con un nombre indígena, mexicano. Varnhagen encontró en el idioma de los huaxtecas las palabras Tamlajab, Tancuayalab y Tancuallalab. Roberto Levillier, que citó este hallazgo de Varnhagen, confirma que estas palabras

$$
\text { "revelan todas analogías con Lariab" (7). }
$$

Se supone, en consecuencia, hasta este momento, que Lariab es una palabra huaxteca oída por Vespucci en la costa de México. Pues bien: se trata de algo muy diferente. Lariab, así escrito por Vespucci, es una palabra china, el nombre de una gran ciudad. Fray Antonio de Remesal, en su Historia de la provincia de San Vicente de Chyaga y Guatimala, nos dice:
"Lamrria, que es la otra insigne ciudad donde estuvo la Corte hasta que echaron a los tártaros de la tierra e hicieron la cerca. Esta es mayor de sitio que Parian. Tiene catorce Tihu, Tichiu diez y siete y noventa y cinco Ticoan" (8).

Lamrria, así escrita, puede haber tenido un sonido suave en la $m$ y otro agudo en la $a$ final y sonar Lariab. Sin otros comentarios, segu:mos con Vespucci.

El segundo viajé comenzó el 16 o 18 de mayo de 1499 . En su carta del 18 de julio de 1500 dice que partió

$$
\text { "par ir a descubrir hacia la parte del noroeste" (9), }
$$

error manifiesto por Sudceste, pues en la Lettera aclara que

"tomamos nuestra navegación por el lebeche" (10),

que significa el viento del sudoeste. Tocó en la costa del Brasil, en los cabos San Agustín y San Roque, y llegó a la desembocadura del

(7). - AMERICO VESPUCIO, El Mundo Nuevo cit. 18.

(8) - CARLOS SANZ, Primitivas relaciones de España con Asia y Oceanía, Madrid, 1958, 287.

(9). - AMERICO VESPUCIO, El Mundo Nuevo cit. 97.

(10). - AMERICO VESPUCIO, El Mundo Nuevo cit. 233. 
Orinoco, de donde siguió viaje a otros puntos y a la isla Española. En la boca del Orinoco,

"un grandísimo río, que es la causa de ser dulce el agua de este golfo", dice que "fuimos a meternos en un golfo, que se llamó el golfo de Parias" (11).

Aparece la palabra o nombre de Parias o Paria, tan repetida, más tarde, durante la conquista.

El nombre de Paria no es indígena. Es palabra china que también se usaba en Filipinas. Los chinos, desde tiempos remotos, tenían comunicación constante con las islas que los españoles llamaron Filipinas. Fray Antonio de Remesal, aún en 1619, atestiguaba que el comercio de los chinos en Manila era intenso y describía sus naves:

"Sus navios son grandes, y no de la forma de Castilla, sin proa, sin bauprés, y sin popa, ni mesana, ni garbias, ni son tan gruesos como los de Castilla, y así ellos llaman a los navios de Castilla Guapangehiou, que quiere decir navio de ancha y gruesa tabla. Y no son breados, sino pegados con halagala, que es un género de betumen muy bueno, y así no huelen mal como los nuestros, no son de la mitad de carga que los de Castilla, aunque tienen la apariencia de muy grandes" (12).

Los chinos eran grandes navegadores. Tenían sus historias y sus mapas. De ellos nos da preciosas noticias fray Antonio de Remesal:

"Tienen libros de la sucesión'de sus reyes, historias de lo que hicieron, y cómo han vivido... Tienen libros de itinerarios de su tierra... Porque también tienen libros de mapas de su tierra pintadas las provincias. $Y$ los trece virreyes que tienen, $y$ los jueces y gobernadores que tienen. En que hay sólo una falta para los curiosos y para poderlos pintar actualmente, y es que no saben de altura de grados, y así se ha de ir a poco más o menos. Porque ya saben de muchas partes a qué altura caen...".

(11). - AMERICO VESPUCIO, El Mundo Nuevo cit. 111.

(12). - FRAY ANTONIO DE REMESAL, Historia de la Provincia de San Vicente de Chyaga y Guatimala, de la Orden de Nuestro glorioso padre Santo Domingo, Madrid, 1619, en CARLOS SANZ, Primitivas relaciones cit. 282. 
Estos navegantes chinos tocaron alguna vez las costas americanas del Pacífico. Las pruebas arqueológicas son conocidas y a ellas nos hemos referido en otras oportunidades. Los contactos de los chinos con los filipinos fueron relatados por Pedro Martir de Anghiera. Este supo por Juan Sebastián de Elcano que en las islas más tarde llamadas Filipinas sus pobladores,

"a más de los dardos del país, de cañas y maderas tostadas, tiene este reyezuelo (de Mactán) picas largas y con hierro, porque recorren aquellas islas los chinos y mercaderes de las regiones chinas".

Miguel López de Legazpi, en una carta al virrey de Nueva España, del año 1572, confirma el hecho, bien transcendente, de que los chinos navegaban con mapas.

"Ellos me dieron una pintura de su costa de los pueblos que vienen a contratar a estas islas que es desde Cantón hasta Lanyicon, la cual juntaron delante de mi sin compás ni orden de altura ni grados, la cual pintura será con esta para que Vuecencia la vea con la relación que se pudo entender de los mismos chinos. .." (13).

Este autor coincide con Legazpi en las características, sin grados, de los mapas chinos y en lo extraordinario de sus navegaciones. Así nos dice que cada año

"van a Parián, que es la Corte, y tierra más fría de cuatro provincias, nueve mil y novecientos y noventa y nueve navíos, cargados de arróz para el rey, y cada navío lleva cinco mil fanegas de las de Castilla, que son cuarenta y nueve millones y novecientos y noventa y cinco mil fanegas" (14).

Ahora sabemos qué significaba y era Parián: nombre de la ciudad principal de China. Remesal nos da más datos:

"La división del reino es esta: Parián, que es ciudad donde está el rey, que está a la banda del Norte, es ciudad toda cercada. Tiene nueve puertas y de puerta a puerta hay más de una legua de camino. Tiene dentro de si una laguna grande, y dos montes de carbón, digo de tierra traída, que se quema como carbón... Tiene el rey la casa en medio de la ciudad, guardadas las puertas

(13). - CARLOS SANZ, Primitivas relaciones cit. 152.

(14). - CARLOS SANZ, Primitivas relaciones cit. 286. 
con elefantes, que es una grandeza extraña, los cuales están siempre trabados por las trompas, y si viene alguno a entrar, alzan las trompas, y si no, no" (15).

Otro misionero, el Padre fray Juan Cobo, atestigua que en Manila el nombre Parián se daba a un gran mercado:

"Estos tienen aquí en Manila junto al río un mercado que llaman Parián, que es una grande cuadra de portales, en medio de los cuales cuatro paños está una balsa de agua grande como una puente al río..." (16).

Es indudable que los chinos no vivían encerrados en su muralla, sino que se extendían por el Pacífico, llegaban a Filipinas y algunas veces tocaban las costas de nuestra América. Fray Domingo de Salazar, primer obispo de Filipinas, escribía a Felipe II, desde Manila, el 24 de junio de 1590 , que

"de todo lo arriba dicho se infiere que todo lo que se ha dicho del reino de China y de los mayores que la gobiernan, en cuanto a no dejar entrar allá gentes extrañas, ha sido invención y embuste de portugueses, que por sus intereses particulares, pensando que su contratación con los chinos había de cesar si entrasen en ella castellanos, que a esto lo hemos atribuído los que acá estamos..." (17).

El nombre de Paria, que Vespucci halló en un golfo de Venezuela -- o él dio a ese golfo - es un nombre chino que correspondía a una ciudad muy importante del imperio y que también se usaba en Filipinas, en Manila, para designar un mercado. La otra ciudad china que seguía en importancia a Parián se llamaba Lamrria, que puede ser Lariab. Vespucci encontró estos nombres en las costas de Venezuela y de México o él dio esas denominaciones a esos lugares. Si se demostrara esto último es indudable que Vespucci conoció esos nonbres por haberlos visto en mapas anteriores al descubrimiento de América o inmediatamente posteriores o por haberlos leído en alguna obra medieval que describía la China. Lo cierto es que el nombre de Paria quedó a la costa de Venezuela y fue usado por los cronistas

(15). - CARLOS SANZ, Primitivas relaciones cit. 286.

(16). - CARLOS SANZ, Primitivas relaciones cit. 161.

(17). - FRAY DOMINGO DE SALAZAR, O. P. Carta relación de las cosas de la China del Parián de Manila. enviada al rey Felipe II por... En CARLOS SANZ, Primitivas relaciones cit. 313. 
que describían esa tierra. También lo emplearon los primeros conquistadores. Vespucci fue el autor o el descubridor de esos nombres - Paria y Lariab -, incuestionablemente chinos, que correspondían a dos grandes ciudades chinas. Vespucci creía estar en las costas del Asia. Lo dice en su carta del 18 de julio de 1500 dirigida a Lorenzo de Pier Francesco de Medici:

"Después de haber nevegado al pie de cuatrocientas leguas continuamente por la costa, llegamos a la conclusión que ésta era tierra firme, como yo digo, y los confines del Asia por la parte de Oriente, y el principio por la parte de Occidente" (18).

Y lo repite al final de su carta:

"... empleando en este viaje trece meses corriendo inmensos peligros, y descubriendo muchísima tierra de Asia..." (19).

Sus propósitos, en un próximo viaje, eran los de hallar la isla de Taprobana, o sea, Ceilán .

"Aquí me arman tres navíos para que nuevamente vaya a descubrir, y creo que estarán listos a mediados de septiembre. Plazca a nuestro Señor concederme salud y buen viaje que a la vuelta espero traer grandes nuevas y descubrir la Isla Taprobana, que se halla entre el mar Indico y el mar Gangético, y después es mi propósito repatriarme y descansar los días de mi vejéz" (20).

Vespucci era cartógrafo y preparó unos mapas para Lorenzo de Médici:

"He resuelto, Magnífico Lorenzo, que así como os he dado cuenta por carta de lo que me ha ocurrido, enviaros dos figuras con la descripción del mundo hechas y preparadas con mis propias manos a saber. Y serán un mapa de figura plana y un Mapamundi de cuerpo esférico, que pienso enviaros por la vía del mar por medio de un tal Francisco Lotti, florentino que se encuentra aquí" (21).

(18). - AMERICO VESPUCIO, El Mundo Nuevo cit. 111.

(19). - AMERICO VESPUCIO, El Mundo Nuevo cit. 120-121.

(20). - AMERICO VESPUCIO, El Mundo Nuevo cit. 122-123.

(21). - AMERICO VESPUCIO, El Mundo Nuevo cit. 123. 
Nosotros hemos planteado el problema de saber si Colón, Vespucci y tantos otros conquistadores que decían recorrer las costas de la India y del Asia estaban en lo cierto o estaban equivocados, engañados por una ilusión geográfica. Esta última suposición es la que ha prevalecido desde mediados del siglo XVI hasta la actualidad. Razones de política, en los pleitos colombinos, y, más tarde, deducciones de geógrafos mal informados, impusieron la creencia de que el continente llamado América era una parte distinta del Asia, que no le correspondía el nombre de India y que Colón y demás exploradores que aseguraban recorrer las costas del Asia vivieron um inmenso error. Nosotros adelantamos la suposición, en 1942, que de que el mapa de Enricus Martellus Germanus, de 1489, mostraba como parte oriental del Asia la masa total de nuestra América (22). Más tarde, en 1970, Dick Edgar Ibarra Grasso demostró definitivamente que Claudio Alejandro Ptolomeo dibujó en su mapamundi el Océano posteriormente llamado Pacífico y las costas americanas que hoy corresponden a Canadá, Estados Unidos, México y demás países del occidente americano (23). Fuimos los primeros en aceptar y confirmar la exposición de Ibarra Grasso. En seguida demostramos que Colón estuvo en lo cierto al decir que su viaje tenía por fin llegar a la India y que, en efecto, tocó la India que Ptolomeo llamaba Oriental y Meridional: la cuarta India, después de las Indias pregangética, gangética y postgangética (24). Ahora bien: Vespucci. lo mismo que Colón, había leído y seguía las enseñanzas perfectas de Ptolomeo, el cual, en su Geografía, enseñaba que al final del Oriente se hallaba el Sinus Magnus, o sea, el olfo Grande, y, junto a él, una tierra - la americana del Pacífico en la cual había una serie de ciudades: Acathra o Acathara, Aspithra, Bramma, Rhabena, Caccorangagara, Thinae, Sarata y Cattigara. También figuraban los ríos Ambastus o Ambato, Soenus y Cuciaris, los promontorios Notium y Satyrarum: todo en el Sinarum Sinus o Golfo de los Sinos.

Vespucci menciona a menudo el nombre de Ptolomeo. Era su guía o mentor permanente. En su carta del 1500, por ejemplo, dice:

"La razón por la cual asigno a cada grado 16 leguas y dos tercios es porque, según Ptolomeo y Alfagrano, la tierra tiene

(22). - ENRIQUE DE GANDLA, Primitivos navegantes vascos, Buenos Aires, 1942, 102.

(23). - DICK EDGAR IBARRA GRASSO, La representación de América en mapas romanos del tiempo de Cristo, Buenos Aires, 1970. passim.

(24). - ENRIQUE DE GANDIA, Claudio Alejandro Ptolomeo cit. 
una circunferencia de 24.000 (millas) que valen 6.000 leguas..." (25).

En su carta del 4 de junio de 1501 vuelve a decir:

"Pero se os dirán así derechamente, como me las contaron, salvo aquello que he corregido un tanto con la cosmografía de Ptolomeo" (26).

Y, en seguida:

"Pues bien sabes cómo Ptolomeo y la mayor parte de la escuela de los cosmógrafos ponen el fin del occidente habitado en las Islas Afortunadas..." (27).

Más adelante, describe el Oriente, según le contó un tal Gaspar, y escribe:

"Creo que sea la provincia que Ptolomeo la llama Gedrosia" (28).

Continúa con el relato y agrega:

"Luego vinieron a una isla llamada Arenbuche, y Malucho, y muchas otras islas del mar Indico, que son las que enumera Ptolomeo, que están alrededor de la Isla Taprobana, y todas son ricas" (29).

Salvo Plinio y algún geógrafo árabe o judío, Vespucci no tiene más consejoros que Ptolomeo.

Nuestro convencimiento de que Ptolomeo conoció, por Marino de Tiro, a quien sigue e intenta corregir, y otros testimonios, la realidad del continente hoy llamado América es absoluto. La mención del río Ambastus o Ambato y de los indios ambatus, que evidentemente son la región y los habitantes de Ambato, en el actual Ecuador y en

(25). - AMERICO VESPUCIO, El Mundo Nuevo cit. 107.

(26). - AMERICO VESPUCIO, El Mundo Nuevo cit. 129.

(27). - AMERICO VESPUCIO, El Mundo Nuevo cit. 129.

(28). - AMERICO VESPUCIO, El Mundo Nuevo cit. 135.

(29). - AMERICO VESPUCIO, El Mundo Nuevo cit. 139. 
otros puntos de Sud América, no puede dejar dudas. El Sinarum Sinus, o Golfo de los Sinos, puede interpretarse como Golfo de los Chinos; pero el nombre de China y de los chinos no fue precisamente éste, sino otro u otros que han variado con el tiempo. Juan Baptista Román, rector de las islas Filipinas en Macán, escribió en una Relación:

"el reino de la China es antiquísimo como se ve por sus anales y corónicas de tantos años atrás y no fue conocido de nuestros antepasados sino de nombre que la llamaron China con poco fundamento a lo menos los chinos no lo saben agora, sino que ellos se llamaron tan antiguamente y el nombre de agora es tamín, costumbres es de la China mudar el nombre del reino y del tiempo cuando se muda el rey de diferente linaje, por lo cual de doscientos años a esta parte se llaman Tamín, por que dura hasta agora que por guerra $u$ otra mudanza se pierde el linaje del primer rey que tomó el renombre para el reino y para el tiempo que durarán sus sucesores cosa muy útil para libros de historias" (30).

El primitivo nombre de la China es ignorado. Puede derivar de Sinus, el Golfo Grande de Ptolomeo, o de la dinastía Tsin, de fines del siglo III antes de Cristo. Lo que a nosotros interesa es que el nombre de Golfo Grande, Sinus Magnus, fue dado por Ptolomeo al hoy llamado Océano Pacífico, que Vespucci leía ávidamente a Ptolomeo y que su fin, al lanzarse a sus navegaciones, fue llegar a este Golfo Grande y, en especial, a la ciudad de Cattigara. Hoy podemos decir que el fin de las navegaciones de Vespucci no fue explorar las inmensidades del Océano Atlántico, sino buscar un paso entre las islas descubiertas por Colón que lo llevase al Sinus Magnus de Ptolomeo y a la ciudad de Cattigara. Hemos repetido el concepto de nuestra tésis porque ella nos revela una verdad, no oculta, sino bien visible, que los historiadores vespuccianos no han querido reconocer.

En primer término hemos comprobado cómo Vespucci afirmó haber tocado en las provincias de Lariab y de Paria y se proponía llegar a la isla de Taprobana. Para llegar a esta isla, en el Océano Indico, nadie ignora que era preciso seguir la costa del Africa y doblar el Cabo de Buena Esperanza o pasar del Atlántico al hoy llamado Pacífico por un estrecho. Pues bien: Vespucci nos revela en su carta del 18 de julio de 1500 cómo era éste su propósito. Son palabras que los comentaristas de Vespucci, por razones inexplicables, no han tenido en cuenta o no han comprendido. Rogamos al lector que las

(30). - CARLOS SANZ, Primitivas relaciones cit. 372. 
lea con detención para saber cómo Vespucci pensaba dirigirse a Cattigara y al Gran Golfo o Sinus Magnus.

"Cuando estuvimos en los navíos, levamos anclas, y nos hicimos a la vela, poniendo proa hacia el mediodía; porque mi intención era ver si podía dar vuelta a un cabo de tierra, que Ptolomeo llama el Cabo Cattegara, que está unido con el Gran Golfo, ya que, mi opinión no estaba muy lejos de ello, según los grados de la longitud y latitud, como se dará cuenta más abajo" (31).

Las palabras de Vespucci no pueden ser más claras y concluyentes. Su intención era dar vuelta a un cabo que Ptolomeo llamaba de Cattigara, unido con el Gran Golfo, o sea, con el Océano hoy llamado Pacífico. El mapa de Claudio Alejandro Ptolomeo nos muestra a Cattigara bien al Sud de la costa del Gran Golfo o Sinus Magnus. Había que poner la proa "hacia el mediodía" para descender cada vez más hacia el Sud, doblar el Cabo de Cattigara, que estaba unido con el Gran Golfo, y de allí navegar derecho hacia Taprobana. Palabra de Vespucci. Y fue así cómo

"navegamos hacia el mediodía y a lo largo de la costa vimos desembocar de la tierra dos grandísimos ríos..." (32).

La navegación, desde este instante, seguirá hacia el Sud.

"Y una vez que hubimos llegado a los navios, levamos anclas haciéndonos a la vela, teniendo continuamente la proa hacia el mediodia" (33).

Hasta que,

"visto el poco camino que hacíamos y el peligro en que estábamos, acordamos volver la proa hacia el maestral y navegar hacia la parte del septentrión" (34).

Tanto habían navegado

(31). - AMERICO VESPUCIO, El Mundo Nuevo cit. 99.

(32). - AMERICO VESPUCIO, El Mundo Nuevo cit. 99.

(33). - AMERICO VESPUCIO, El Mundo Nuevo cit. 101.

(34). - AMERICO VESPUCIO, El Mundo Nuevo cit. 101. 
"hacia la parte del mediodía que entramos en la zona tórrida y dentro del círculo del Cáncer..." (35).

Nuestra demostración, bien simple, con las propias palabras de Vespucci, de que su propósito era-navegar hacia el Sud, hasta doblar el Cabo de Cattigara y llegar a la isla de Taprobana, se halla confirmada, amplísimamente, por su otra carta del 4 de junio de 1501, escrita desde Cabo Verde a Lorenzo de Pier Francesco de Medici. En ella le refiere que en la isla de Cabo Verde se encontró con la armada de Pedro Alvarez Cabral, que había tocado en el Brasil y seguido viaje a la India. Un tal Gaspar, que había hecho dos veces el viaje de Portugal al Mar Indico, le dijo que

"en el interior de la costa del Mar Pérsico"

había muchas ciudades, una de las cuales se llamaba "Torsis" (36). Muchas cosas le contó este Gaspar. En la ciudad de Emparlicat, por ejemplo,

"está el cuerpo del apostol San Marcos y hay muchos cristianos" (37).

Gaspar había recorrido la isla de Ceilán,

"riquísima de piedras preciosas y de perlas, y de especiería de todo género, y de droguería y otras riquezas, como son elefantes y gran caballería; de modo que estimo que ésta sea la Isla Taprobana, según él me la pinta".

Gaspar había estado asimismo

"en otra isla que se llama Sumatra, la cual es de tanta extensión como Ceilán y Bencomarcano, a la vez que es tan rica como ellas; de modo que no siendo Ceilán la Isla Taprobana, será Sumatra. De estas dos islas vienen a Persia y Arabia infinitos navíos cargados de todo género de especiería y droguería y piedras preciosas" (38).

(35). - AMERICO VESPUCIO, El Mundo Nuevo cit. 101.

(36). - AMERICO VESPUCIO, El Mundo Nuevo cit. 135.

(37). - AMERICO VESPUCIO, El Mundo Nuevo cit. 139.

(38). - AMERICO VESPUCIO, El Mundo Nuevo cit. 139. 
Todo esto oyó Vespucci de este famoso Gaspar que tanto había andado por el Oriente. Estas y otras maravillas que Vespucci enumera en esta carta reveladora, que llenó su mente de fantasías. Y todo esto fue lo que Vespucci se propuso alcanzar y descubrir en el viaje que estaba por emprender o, mejor dicho, que ya había emprendido rumbo a las regiones del Sud, al final de la tierra que había empezado a recorrer, como había contado en su carta del año 1500 , y que había debido interrumpir por los peligros y volver hacia el Norte. Esta vez no le ocurriría lo mismo: iría en busca de Taprobana por el camino del cabo de Cattigara, como había explicado en su carta del año 1500 , y vería las tierras asombrosas que había visitado su amigo Gaspar. No somos nosotros quienes decimos esto, quienes revelamos el itinerario de su viaje de 1501 y 1502 . Es el proprio Vespucci quien nos lo dice con palabras seguras y firmes, que sus comentaristas no han sabido o querido escuchar. He aquí las palabras de Vespucci:

"Y yo tengo esperanza, en esta navegación mía, volverlas a ver y recorrer gran parte de lo antedicho, y descubrir mucho más, y a mi vuelta haré de todo buena y verdadera relación" (39).

El viaje de 1501 y 1502 está, por tanto, delineado, prefijado, establecido, con una meta cierta, con un fin y un destino perfectamente definidos, en esta carta de 1501 . Vespucci, en el viaje que ya había emprendido, como dice en esta carta escrita en el Cabo Verde,

"que es el principio de la provincia de Etiopía y está en el meridiano de las Islas Afortunadas",

se proponía ir a las regiones adónde había ido Pedro Alvarez Cabral y, en particular, a la isla de Ceilán o Taprobana, adonde había llegado su amigo Gaspar. Si Ceilán no era la Taprobana de Tolomeo llegaría a la isla de Sumatra. Ahora bien: para llegar al hoy llamado Oceáno Pacífico, es decir, al Sinus Magnus de Ptolomeo, para alcanzar ese Océano Indico adonde había llegado Pedro Alvarez Cabral, para descubrir las riquezas de Ceilán y de Sumatra, no había, por agua, más que dos caminos en el mundo: el del Cabo de Buena Esperanza o el del hoy llamado Estrecho de Magallanes. Cabral había sido por el Cabo de Buena Esperanza. Vespucci iría por el hoy llamado Estrecho de Magallanes, por el Cabo de Cattigara. Doblaría ese Cabo de Cattigara, tan mencionado por Ptolomeo, entraría en el Sinus Magnus - hoy Océano Pacífico - y allí buscaría la isla de Ceilán o la de Sumatra, o sea, la antigua Taprobana de los geógrafos antiguos.

(39). - AMERICO VESPUCIO, El Mundo Nuevo cit. 137. 
Este es el itinerario que Vespucci se propuso seguir en su viaje de 1501 y 1502 . Está explicado, muy bien dicho, por él, en sus cartas de 1500 y 1501 . Algunos historiadores, empeñados, por motivos inexplicables, en negar este viaje de Vespucci todo a lo largo de la costa patagónica, lo han querido apartar del Brasil, en un determinado momento, y enviar Océano afuera, al azar, hacia lo desconocido, a explorar la inmensidad del mar, como un insensato o un loco, sin una meta preestablecida, hasta dar con un témpano o una ballena en el paralelo 50 o 52, que él habría confundido con una tierra, y desde donde o desde la cual se habría vuelto a España. También, para probar que no había seguido la costa en busca de un paso - el Cabo de Cattigara - que le permitiera pasar al otro mar, al Sinus Magnus de Ptolomeo, estos historiadores han asegurado que Vespucci no podía violar la línea de Tordesillas, que estaba obligado a respetarla, a no seguir la costa patagónica, que debía obedecer el tratado hsipano-portugués y navegar mar adentro, bien lejos de la línea de Tordesillas y, por tanto, de la costa luego llamada patagónica. No mencionamos los nombres de quienes han querido, alguna vez, discutirnos estos puntos. Los lectores saben, definitivamente, que Vespucci partió, en su via e de 1501-1502, en busca de un paso al Sinus Magnus de Ptolomeo, el Cabo de Cattigara, para alcanzar las riquezas fabulosas de la isla Taprobana, la Ceilán o la Sumatra maravillosas de las cuales tanto le había hablado el sorprendente Gaspar.

Llegados a este punto debemos decir algo de Cattigara. Su historia está en Marino de Tiro y en Claudio Alejandro Ptolomeo. Sabemos por el vizconde de Santarem, muy ilustre investigador brasileño, desde el 1849 , que Marino de Tiro fue leído por el geógrafo árabe Almasudi. Este geógrafo nació en Bagdad, vivió diez años en Siria y Egipto y murió en el Cairo en el año 957 de nuestra Era. Almasudi dice que vio

"los climas iluminados con diversos colores en varios libros"

y lo meior de este género en el Tratado de Geografía de Marino de Tiro. Este Tratado se ha perdido; pero mucho de él ha llegado hasta nosotros a través de Claudio Alejandro Ptolomeo, el "príncipe de los astrónomos", que vivió en Alejandría, en Egipto, en tiempos del emperador Adriano Antonino, en la primera mitad del siglo II de nuestra Era. Su Geografía fue leída en griego y en latín hasta el año 1548 en que se tradujo por primera vez al italiano. No se sabe exactamente si Ptolomeo dibuió su mapamundi. El más antiguo manuscripto que se conserva es del siglo XII o XIII. El mapamundi pudo ser dibujado por Agathodiamon de Alejandría, probablemente contemporáneo de Pto- 
lomeo. Ptolomeo no sabía con precisión dónde se hallaba Tiro, en la costa fenicia. Recuerda que Marino consultó los autores que lo precedieron en tiempos remotos. Eran, sin duda, los geógrafos helenísticos que describían los caminos al Oriente y componían mapas como los que menciona Plutarco. En los siglos helenísticos, los mercaderes romanos navegaban, impulsados por los monsones, desde el Mar Rojo hasta la India, Malaya y la Indochina. Otros seguían por tierra la vieja ruta de la seda, hasta los mercados de Pamir, donde traficaban con los indios y chinos. El geógrafo griego Cosmas Indicopleustes explicó que podía llegarse a Tzinitza más rápidamente atravesando Persia que por mar. Los dos caminos al Oriente - por tierra y por mar - fueron cerrados por los árabes en el siglo VII. Ptolomeo, por tanto, sabía por Marino de Tiro y otras fuentes que el Oriente era explorado desde siglos por muchos viajeros. Decía que en tiempos de Marino de Tiro ciertas estrellas, como el Perro o Can y el Anticán, estaban en Tauro y en Géminis, por lo cual,

"antes que el Sol tocase el Cáncer, salían por la mañana de entre los rayos; pero ahora es diferente".

Corrigió a Marino de Tiro en la medida de la anchura terrestre. En el Libro I, capítulo X, sotuvo que lo ancho de la Tierra no era de 87 grados, o sea, 43.500 estadios, como afirmaba Marino, sino de 80 grados o 40.000 estadios. En el Libro I, capítulo XI, puso el fin de Occidente en las Islas Afortunadas, y el del Oriente,

"en las crueles y horrendas partes de Sera y de Sina e igualmente de Cattigara".

Todo ésto fundado en Marino de Tiro. Cattigara era, en consecuencia, según Marino de Tiro y Ptolomeo, la ciudad más lejana del mundo. Según Marino, el viaje desde la Torre de Piedra, por donde pasaba el Eufrates, siguiendo ese paralelo por Hierópolis y Rodas, hasta Sera, ciudad principal de los Seris, duraba siete meses. El viaje debía hacerse por los paralelos que cruzaban el Helesponto y Constantinopla. En el trayecto había "cruelísimas tempestades". Marino afirmaba conocer esos viajes por mercaderes de Macedonia. A su juicio, entre las Islas Afortundas y Sera había 225 grados. Ptolomeo reducía estos grados a 180 . Según Marino, entre la Torre de Piedra y Sera había 36.200 estadios, y según Ptolomeo, 22.625 estadios, o sea, 45 grados y un cuarto, correspondiendo 500 estadios por grado. Sin las vueltas, la distancia entre el Eufrates y Sera era de 105 grados y un cuarto. Concluía sosteniendo que la distancia universal de la Tierra desde las Islas Afortunadas era de 177 grados y un cuarto. 
Ptolomeo describe el viaie hasta la India y el Auro Quersoneo en el Libro I, capítulo XIII. En el Libro I, capítulo XIV, estudia

"la navegación que se hace desde el Aurea Península hasta Cattigara".

Ptolomeo discute la navegación indicada por Marino de Tiro. Deduce que la distancia desde la Península Aurea hasta Cattigara es de 17 grados y 10 minutos y concluye que el ancho universal de la tierra conocida contiene 180 grados, o sea, 12 intervalos de hora. En el Libro I, capítulo XVII, vuelve a corregir a Marino de Tiro. A su entender, la duración de la navegación "es irregular y desordenada". No acepta la ruta señalada por Marino de Tiro desde la ciudad principal de Sina hasta el puerto de Cattigara. En la Tabla XI del Asia menciona las ciudades principales de la India, fuera del Ganges: Apithra, Sina y Cattigara. En el Libro VII, capítulo III, habla de la ciudad de Thina.

"Esta ciudad dicen que no tiene las murallas de metal ni otra cosa digna de memoria. Está circundada por los cattigaros y hacia occidente por la tierra incógnita que abraza el Mar llamado Praso, hasta el Promontorio de Praso, donde empieza el Mar áspero que une la tierra con el Promontorio Rapto y con las partes australes de Azania".

En el Libro I, capítulo XIV, menciona a Alejandro, el cual "escribió" el itinerario a Cattigara. Primero había que ir a Zaba (según Dick Edgar Ibarra Grasso podría ser Sumatra)

"y desde Zaba, navegando hacia el Austro y más hacia la banda izquierda, en algunos días llegan a Cattigara".

Marino explicó que por ser muchísimos los días empleados en esa navegación no fueron numerados. Ptolomeo piensa que "algunos días" equivalen a unos pocos días. Así habla Ptolomeo:

"Marino además no explica el número de los estadios de aquella navegación que se hace de Aurea Península a Cattigara, pero dice que Alejandro escribió que la tierra por la cual se camina está opuesta al medio día y que los que navegan cerca de ella llegan en veinte días a la ciudad llamada Zaba y de Zaba navegando hacia el Austro y más hacia la banda izquierda en 
algunos dias llegan a Cattigara. Aunque por ello dice que por su multitud no fueron enumerados".

Ptolomeo opina que el meridiano que pasa por Cattigara dista de las Islas Afortunadas 177 partes. Hacia el Sud, Cattigara está en $8^{\circ}$ y 1/2. De Aurea Quersoneso a Cattigara pone una distancia de 17 grados y 10 minutos. En el Libro I, capítulo IX, Ptolomeo habla de un Diógenes que había navegado en la India, y de un Teófilo que navegaba en Azania. Cattigara aparece, junto con otras ciudades, en las tablas de la Geografía de Ptolomeo en $8^{\circ} 30$ de Latitud Sud, sobre la costa oriental del Sinus Magnus. Al Norte figuran los Ambatos, incuestionablemente los indios ambatos del Ecuador, y la ciudad de Ambato.

Cattigara ha sido identificada en el siglo XVIII con Cantón. Otros autores la han colocado en Indochina y confundido con Bangkock, gran puerto de Tailandia. En la costa occidental de la isla Bohoi, una de las Filipinas, tenemos un pueblo llamado Catigbian. En un mapa anónimo de la escuela de Reinal, tal vez del año 1519, se ve un rastro del Sinus Magnus junto a la isla de Ceilán y enfrente la inscripción: Camatara. En el planisferio de Alberto Cantino, agente secreto de Hércules d'Este, duque de Ferrara, probablemente de la segunda quincena de octubre de 1502 . hecho por un portugués desconocido, aparece en el golfo de Bengala la ciudad de Carigan, la Chatigan de los portugueses y la Chittagong de los ingleses, también llamada, respectivamente, Sategán y Satgaon. La búsqueda de nombres de poblaciones o regiones parecidos a Cattigara encuentra similitudes; pero no coincidencias indiscutibles ni explicaciones. Sólo en el idioma guaraní hemos hallado, con exactitud, el significado de Cattigara. Cati significa hediondo, olor fuerte, e ivirá es el nombre de un árbol tintoreo. Por ello en la Argentina existe el nombre Catigua. En Bolivia hay Capiguara, de capi-ivá, carpincho (cepi-í, paja, y guara, morador: un animal que vive entre la paja). En el Brasil hay catiguá, de cáa, planta, y guá, rayado, pintado. En Chile existe Capibara, del guaraní capibara, carpincho, que vive entre la hierba. En Venezuela se conoce capivara, de capí-ivá, carpincho. El el Paraguay es común capiguara, de capi-í, espadaña, hierba, y guara, habitante, o de capí-ivá, carpincho, y ujhara, comedor, comedores de carpinchos. También de capi-í, espadaña, e iguá, laguna: espadaña de aguada (40). Además, en nuestros estudios sobre la conquista del Paraguay hemos hallado un cacique principal "que se dice Cotiguara" y que

(40). - A. JOVER PERALTA, El guaraní en la geografía de América: Diccionario de guaranismo. Editorial Tupá. Buenos Aires, 1950. 
dio mucho que hacer a los españoles (41). Cattigara podría ser un nombre guaraní, con el significado de Hombres que viven entre la hierba u otro más o menos equivalente. El significado preciso no interesa y podran deducirlo los etimologistas. También podría ser explicado por medio del sánscrito. Lo probable es que sea palabra de origen guaraní. Muy bien recuerda A. Jover Peralta una opinión del eminente americanista Samuel A. Lafone Quevedo:

"No me atrevo a unir léxicamente el araucano con el guaraní; pero existen ciertas afinidades que no deberían pasarse por alto, y la palabra co, que significa agua, es común a la región araucana y la brasiliensi. Si Montesinos no inventa, hordas caríbicas invadieron los Andes, $y$ su presencia en la región de los sufijos puede haber modificado el vocabulario local".

No insistimos sobre el origen guaraní del nombre Cattigara, en el Pacífico, adonde llegaron, indiscutiblemente, grupos guaraníes y dejaron sus denominaciones en infinidad de lugares. Tampouco buscamos las etimologías de otros nombres de localidades y ríos de la costa peruana señalados por Ptolomeo. Thinae o Thine, por ejemplo, puede emparentarse con Tinian y Tahití, islas marianas, en el Pacífico.

Un hecho cierto es que los viajes al Oriente, desde el Mediterráneo, databan de la época helenística. Marino de Tiro los estudió en su obra perdida. Ptolomeo apenas reprodujo sus pasajes más notables, discutiéndolos en detalles donde no tuvo razón. La barrera de los árabes no impidió las comunicaciones con el Asia lejana. El atlas catalán de 1375, dibujado por el judío mallorquín Abraham Cresques, cartógrafo del rey de Aragón, fue presentado al rey de Francia Carlos V en 1381. El el más hermoso y completo de todos los mapas medievales. Se halla original en la Biblioteca Nacional de París, manuscripto español número 30 , y muestra las caravanas que iban desde Bokhara hasta Pekín. El ansia de hallar una nueva ruta en el Oriente era el clima que hizo posibles los intentos portugueses de circunnavegar el Africa y alcanzar el dominio de la India. El relato de Marco Polo, dictado a Rusticello da Pisa, en la prisión de Génova, en 1298, enloqueció a Europa. Hablaba de Kinsay, capital de Manji, como

"la más noble y rica ciudad del mundo".

(41). - ENRIQUE DE GANDIA, Historia de la conquista del Río de la Plata y del Paraguay, Buenos Aires, 1931, 141. 
Zaytón, el gran puerto de Manji, era

"uno de los dos más grandes puertos del mundo por el monto de su comercio".

Kinsay era la ciudad del cielo. Tenía cien millas de circuito y estaba construída como Venecia, con canales, doce puertas y doce mil puentes. Es curioso que Polo no menciona la muralla china, ni el té, ni las características de la lengua china. Sus fechas no coinciden con otras bien conocidas. El Millón se multiplicó en cientos de manuscriptos. Se conocen ciento treinta y ocho. Antes del año 1500 fue traducido al latín, italiano, alemán y español. Colón lo leyó. La primera traducción de Polo al español fue hecha por Rodrigo de Santaella en agosto de 1493 y se publicó en 1505 . Después de Polo, Juan de Monte Corvino, primer arzobispo de Pekin, vivió en China desde 1293 hasta 1328. El hermano Oderico de Podernone visitó la India entre 1316 y 1330 . Llegó a Cantón, pasó tres años en Pekin y regresó a Europa por el Tibet y Persia. Juan Marignoli vivió en Pekin como legado del Papa desde el 1342 hasta el 1346. En 1368 los mongcles pusieron fin a la dinastia de los Ming y los turcos cerraron la ruta al Cathay. China empezó a ser un gran mistero; pero la esperanza del Africa, que el faraón Neco había hecho circunnavegar por los fenicios seiscientos años antes de Cristo, según Herodoto, convencía a los portugueses de que podían doblar su extremo Sud y llegar a la India. En 1488, Pedro de Covilhão fue el primer portugués que cruzó el Egipto y el Mar Rojo y llegó a la India. Estuvo de vuelta en el Cairo en 1490 y escribió al rey Juan que había visitado las ciudades de Cananor, Calicut y Goa. Luego se fue a Abisinia y allí lo encontró una embajada portuguesa en 1520 . Un año autes había doblado el Cabo de Buena Esperanza Bartolomé Días, en su intento de llegar a la India. Volvió en 1489 y en seguida el geógrafo Enricus Martellus Germanus consignó los viajes de Cão y de Días en su gran mapamundi de 1489 .

En el siglo XV aparecieron los primeros mapas impresos. Hay de ellos unos trescientos grabados en madera o en cobre. Los más atrayentes fueron los de Ptolomeo, impresos en Bolonia en 1477. De las primeras cinco ediciones quedan unos trescientos treinta y dos ejemplares. Vasco de Gama fue el primero que llegó a Calicut en mayo de 1498 doblando el Cabo de Buena Esperanza. El planisferio de Alberto Cantino, dibujado por un anónimo portugués, en octubre de 1502, tiene en el Brasil una inscripción que dice que esa tierra la descubrió Pedro Alvarez Cabral 


\begin{abstract}
"que o dito Rey mandava a Caliqut y en el caminho indo topou com esta terra em a qual terra se cree se terra firme".
\end{abstract}

El rey de Portugal había enviado a Alvarez Cabral a Calicut por la vía del Occidente y no pudo pasar del Brasil por no encontrar un paso. Había que hallar este paso y Vespucci, que no ignoraba todo cuanto hemos expuesto, sabía que el paso existía, no en la masa del Brasil, sino mucho más al Sud. Y lo sabía por el mapamundi de Ptolomeo, donde aparecía Cattigara al final de la tierra oriental de los indios ambatos, cattigaras y otros, y por el mapa de Enricus Martellus Germanus, de 1489, que dibujaba la parte oriental de la cuarta India, copiándola de Ptolomeo, con el Sinus Magnus ptolemáico, y la parte occidental, hasta entonces desconocida, pero impresionante en su parecido con la realidad atlántica.

Hemos mencionado a Enricus Martellus Germanus. De él poco se sabe. Dibujó su mapamundi Insularium en Florencia, en 1489, consignando el viaje de Bartolomé Días. Su mapamundi, sorprendente por su revelación de todo el continente hoy llamado americano, se encuentra en el Museo Británico de Londres y tiene la asigratura Add. MS. 15760. La tierra meridional, que unía el Africa con el Sud Este del Asia, o sea, de la América del Sud, como figuraba en el mapamundi de Ptolomeo y de sus repetidores, desaparece. El Este del Asia tiene la configuración del continente americano. Este mapa fue copiado, con el agregado de muchas islas en el Atlántico, por Martín Behaim o de Bohemia, en 1492, poco antes de conocerse en Europa el resultado del viaje de Colón. Lo sorprendente de Enricus Martellus Germanus es, repetimos, que el Este del Asia es la gran masa americana y que ésta tiene al final de lo que hoy llamamos Patagonia y costas de Chile, un estrecho y el triángulo del archipiélago fueguino. Los nombres que lo circundan son de regiones asiáticas. Martellus no sabía dónde colocarlos y los diseminó en lugares que no les corresponden.

Nadie ignoraba, en España y en Portugal, al igual que en el resto de Europa, que se podía llegar a la India por la vía del Mar Rojo o del Cabo de Buena Esperanza y por la vía del paso de Cattigara o del estrecho dibujado en los mapamundis de Enricus Martellus Germanus y de Martín Eehaim. El rey de Portugal envió en 1498 a Duarte Pacheco a descubrir las regiones del Oeste; pero no lo hizo por saber que esa gran masa de tierra no tenía un estrecho o paso que le permitiese llegar al otro mar, al Sinus Magnus de Ptolomeo, y la India gangética. Es en estos momentos que Vespucci emprende sus viajes, El primero lo llevó a regiones que llamó La- 
riab y Paria, nombres de provincias de la China. El segundo fracasó en su intento de dirigirse hacia el Sud. En ese tiempo tuvo conocimiento del viaje de Cabral a la India, supo las maravillas del Asia por los relatos de Gaspar y se lanzó, en la expedición de Gonzalo Coelho, resuelto a doblar el cabo de Cattigara, dibujado por Ptolomeo, y navegar hasta las regiones descriptas por Gaspar. Así lo dice, como hemos leído, el propio Vespucci en sus cartas a Lorenzo de Médicis. Su intento de pasar al Sinus Magnus, el otro mar dibujado por Ptolomeo, había comenzado con el mismo Cristóbal Colón, según refieren su hijo don Hernando y otros cronistas, y había sido repetido por Juan Caboto, que partió en busca del Catayo y se detuvo en Nueva Escocia, en la América del Norte, convencido de encontrarse en el Asia, y por Gaspar de Corte Real, de las Azores, que redescubrió Groenlandia y llegó al Labrador entre 1500 y 1501 .

Américo Vespucci, en su carta del 4 de junio de 1501, escrita en el Cabo Verde, dijo a Lorenzo de Médici que el viajero Gaspar le había contado maravillas del Asia y

"yo tengo esperanza en esta navegación mía volverlas a ver, y recorrer gran parte de lo antedicho, y descubrir mucho más, y a mi vuelta haré de todo buena y verdadera relación" (42).

En su carta siguiente, del año 1502 , escrita probablemente desde Lisboa, se refiere a su carta del 4 de junio de 1501 y le dice:

"La última escrita a Vuestra Magnificencia fue desde la costa de Guinea, desde un lugar que se llama el Cabo Verde, por la cual supisteis el principio de mi viaje, y por la presente se os dirá brevemente el medio y el fin de él, que es cuanto sigue ahora" (43).

Estamos al comienzo del discutido viaje de 1501-1502. Podemos seguirlo con cuatro testimonios del propio Vespucci: su carta de 1502, la carta llamada fragmentaria escrita entre septiembre y diciembre de 1502, El Nuevo Mundo, tal vez de 1503, y la carta o Lettera, en que resume sus cuatro viajes.

En su carta de 1502 dice que se adentraron (44)

"en el golfo del mar Océano para buscar nuevas tierras"

(42). - AMERICO VESPUCIO, El Mundo Nuevo cit. 137.

(43). - AMERICO VESPUCIO, El Mundo Nuevo cit. 143.

(44). - AMERICO VESPUCIO, El Mundo Nuevo cit. 143. 


\section{En El Nuevo Mundo repite que}

"con feliz nevegación, a 14 días del mes de mayo de 1501, partimos de Lisboa, por orden del mencionado rey, con tres naves a buscar nuevos países hacia el austro, y navegamos veinte meses continuamente hacia el medio día" (45).

\section{En la Lettera confirma que el rey de Portugal}

"me rogó que fuese en compañia de tres de sus naves que estaban prestas para ir a descubrir nuevas tierras" (46).

Partieron de Lisboa el 10 de mayo de 1501. En El Nuevo Mundo había dicho el 14 de mayo.

En la carta de 1502 refiere que

"viajamos tanto por estos mares, que entramos en la zona tórrida, y pasamos la línea equinocial por el lado del austro, y del trópico de Capricornio, tanto que el polo del mediodía estaba arriba de mi horizonte 50 grados, y otro tanto con mi latitud de la línea equinocial, y navegamos cuatro meses y veintiseis días..." (47).

Hemos mostrado el primer testimonio en que Vespucci asegura que alcanzó los cincuenta grados de Latitud Sud. Su carta de 1502 se pierde en descripciones de la vida de los indígenas. En su carta fragmentaria, también de 1502, aclara que

"Verdad es que cuando llegamos a la altura de los 50 grados estábamos en el mar y no en tierra, porque cuando nos apartamos da la tierra no estábamos a mayor altura que a 32 grados y luego tanto navegamos por el viento siroco que fuimos a la dicha altura de 50 grados y sin tierra, aunque juzgábamos estar cerca de tierra por muchas señales que veíamos, que fueron infinitos pájaros de diversas clases y muchos maderos en el mar: señales ciertísimas" (48).

En la misma carta hace otra aclaración:

"Y el continente de la tierra por mi vista y navegada, se encierra entre estos dos trópicos, o por mejor decir parlelos, el uno

\footnotetext{
(45). - AMERICO VESPUCIO, El Mundo Nuevo cit. 173.

(46). - AMERICO VESPUCIO, El Mundo Nuevo cit. 249.

(47). - AMERICO VESPUCIO, El Mundo Nuevo cit. 145.

(48). - AMERICO VESPUCIO, El Mundo Nuevo cit. 155.
} 
que está en la región septentrional distante de la línea equinocial 33 grados, el otro hacia el austro que dista de dicha línea 32 grados, y no piensen por un solo meridiano, sino por diversos..." (49).

Aquí encontramos un error, gravísimo, que es preciso rectificar. Los dos trópicos a que se refiere Vespucci - el del Norte y el del Sud - no están en 33 ni en 32 grados de Latitud Norte y Latitud Sud, sino en 23 grados 27 minutos de las dos latitudes. Ahora bien: consta que Vespucci sabía la latitud de los trópicos, como la sabía cualquier marino de insignificantes conocimientos. En la misma carta fragmentaria vuelve a decir:

"Porque como antes he dicho, yo he navegado por todos los paralelos que hay desde el Marruecos hasta el fin de Etiopía y pasando la línea equinocial 32 grados hacia el austro" (50).

Veremos cómo no navegó hasta 32 grados de latitud Sud, como aquí parece decir, sino hasta los 52 grados de la misma latitud. No sabemos a qué se debe este error con el número 32. Es el propio Vespucci que lo corrije y expresa que el trópico se hallaba en 23 grados y minutos y él había navegado hasta los $\mathbf{5 0}$ grados:

"en cuanto a aquello que me preguntas si con mi cenit yo había pasado el trópico del Capricornio, la carta lo demuestra; ya que el trópico dista de la línea equinocial 23 grados y $51 \mathrm{mi-}$ nutos, y yo fui hasta 50 grados próximos al círculo antártico" (51).

No hay duda, por tanto - y no pueden haber discusiones - de que el párrafo anterior referente a los trópicos y a su navegación hasta los 32 grados de Latitud Sud contiene dos gruesos errores.

En El Nuevo Mundo, Vespucci refiere a Lorenzo de Médici que, una vez en la costa hoy llamada americana,

"navegamos siguiendo el litoral cerca de seiscientas leguas, y muchas veces descendimos a tierra y hablábamos y nos comunicábamos con los del país..." (52).

(49). - AMERICO VESPUCIO, El Mundo Nuevo cit. 157.

(50). - AMERICO VESPUCIO, El Mundo Nuevo cit. 159.

(51). - AMERICO VESPUCIO, El Mundo Nuevo cit. 163.

(52). - AMERICO VESPUCIO, El Mundo Nuevo cit. 179. 
Hemos leído que Vespucci y su gente siguieron el litoral cerca de seiscientas leguas y cada tanto bajaban a tierra y hablaban con los naturales. No dice que se haya apartado de la costa, que haya salido mar afuera, que abandonara esa tierra en los 32 grados de Latitud Sud y corrieran, las tres naves, por el Océano infinito, sin rumbo, sin saber adónde iban ni qué buscaban. Esto no consta en este documento. Por el contrario: dice esto otro:

"Siguiendo esta playa tan largo tiempo navegamos que pasado el trópico de Capricornio encontramos el polo antártico' en su horizonte más alto 50 grados, y estuvimos cerca de ese círculo antártico en los 17 grados y medio" (53).

He aquí la prueba definitiva, indiscutible, de que es el propio Vespucci quien dice que, después de haber navegado seiscientas leguas "siguiendo el litoral", descendiendo a tierra y hablando con la gente del país, navegaron "siguiendo esta playa tan largo tiempo" que llegaron a los 50 grados. No dice que se apartaran de la casta ni que navegaran mar afuera. Repetimos: "siguiendo el litoral" seiscientas leguas e, inmediatamente, "siguiendo esta playa" hasta los 50 grados de Latitud Sud. Más claro, imposible.

La llegada de Vespucci hasta los 50 grados de Latitud Sud está confirmada en otro párrafo de El Nuevo Mundo, donde dice:

"Pues bien, como te he dicho, desde Lisboa donde nosotros partimos, que de la línea equinocial está distante 39 grados y medio, y navegamos más allá de la línea equinocial por 50 grados..." (54).

Desde Lisboa, que se hallaba en 39 grados y medio, había navegado hasta los 50 grados de Latitud Sud. Es concluyente. Y también es aceptable la suposición de que en los 50 grados de Latitud Sud, adonde él había llegado, habitaban seres humanos. Así lo dice en otro párrafo:

"Y por esta rzón nosotros los que habitamos Lisboa cerca de la línea equinocial 39 grado y medio de Latitud septentrional, estamos ".cima de aquellos qut 'abitan a los 50 grados de latitud meridional..." (55).

(53). - AMERICO VESPUCIO, El Mundo Nuevo cit. 179.

(54). - AMERICO VESPUCIO, El Mundo Nuevo cit. 191.

(55). - AMERICO VESPUCIO, El Mundo Nuevo cit. 191. 
Rogamos que se recuerden estas dos latitudes - 39 grados y medio de Lisboa y 50 grados de latitud meridional - y se compruebe cómo está equivocado el párrafo de la carta llamada fragmentaria, escrita entre septiembre y diciembre de 1502, donde dice que

"Al co. inente de la tierra por mi vista y navegada, se encierra entre tstos dos trópicos, o por mejor decir, paralelos, el uno que está en la región septentrional distante de la línea equinocial 33 grados, el otro hacia el austro que dista de dicha línea 32 grados...".

En El Nuevo Mundo habla de 39 grados y medio Norte, Lisboa, y de 50 grados de Latitud Sud, y en la carta fragmentaria de $\mathbf{3 3}$ grados Latitud Norte y 32 grados de Latitud Sud: errores innegables. Esta carta fragmentaria, con semejantes errores, es la que esgrimen algunos polemistas para insistir en que Vespucci sólo navegó en la costa americana hasta los 32 grados de Latitud Sud. Vespucci se equivocaba en fracciones de grado. Es sabido, por ejemplo, que Lisboa no está precisamente en 39 grados y medio, sino en algo menos de 39 grados.

Todo lo expuesto por Vespucci en sus cartas manuscriptas es confirmado por la Lettera, pero en ella, como documento impreso, se han deslizado algunos errores. En la Lettera nos dice que

"así navegamos tanto que encontramos que la costa daba vuelta hacia el lebeche, y doblando un cabo al que pusimos por nombre Cabo de San Agustín, comenzamos a navegar hacia el lebeche",

es decir, hacia el Sudoeste (56). El Cabo de San Agustín se hallaba en 8 grados

"fuera de la línea equinocial, hacia el austro" (57).

Continúa:

"Tanto navegamos hacia el austro que ya estábamos fuera del trópico de Capricornio, donde el polo del mediodía se alzaba sobre el horizonte 32 grados y ya habíamos perdido completamente

(56). - AMERICO VESPUCIO, El Mundo Nuevo cit. 255.

(57). - AMERICO VESPUCIO, El Mundo Nuevo cit. 255. 
la Osa Menor, y la Mayo. staba muy baja, y apenas aparecia en la línea del horizonte..." (58).

Otra vez un error indudable: $\mathrm{Si}$ los 32 grados se refieren al trópico del Capricornio, es sabido que el trópico está en 23 grados y minutos, y si se refiere al punto máximo alcanzado en aquella navegación también sabemos que fue de 52 grados. Vespucci agrega, como explicación, que

"recorrimos al pie de 750 leguas de esta costa: 150 desde el dicho Cabo de San Agustín hacia el poniente, y 600 hacia el lebeche..."

o sea, hacia el Sudoeste (59).

Vespucci y la demás gente

"en esta costa no vimos cosa de provecho, excepto innumerables árboles de brasil y de casia y de los que producen la mirra u otras maravillas...".

Es incuestionable que Vespucci y su gente seguían la costa sin apartarse de ella. Buscaban riquezas. Al cabo de diez meses,

"visto que en esta tierra no encontrábamos ninguna mina, acordamos despedirnos de ella e ir a explorar el mar por otra parte. Hecho nuestro consejo se resolvió que se siguiese aquella navegación que me pareciera bien, y fue puesto en mí todo el mando de la flota".

Vespucci ordenó que se hiciesen provisiones de agua y leña para seis meses,

"pues ese tiempo estimaron los oficiales de las naves que podríamos navegar en ellas" (60).

Recapitulemos: Vespucci, al comprobar que no encontraban ninguna mina, tomó el mando de la expedición. Era el jefe. Estaba encargado de llevarla adonde "me pareciera bien". Recordemos cuál era su meta, el fin supremo de sus ilusiones, y lo que nos dijo en sus cartas: el Sud, doblar el cabo de Cattigara, señalado por Ptolomeo,

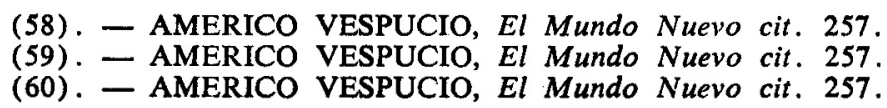


buscar en el Sinus Magnus, en el otro mar, en el Indico, la isla de Taprobana o Ceilán, donde había infinitas riquezas. De ellas le había hablado el fantástico Gaspar. El paso estaba dibujado en los mapamundis de Enricus Martellus Germanus y Martín Behaim. Hizo provisiones para seis meses. Comenzó su navegación "por el viento siroco", el 15 de febrero de 1502.

"Y tanto navegamos por este viento que nos encontramos tan altos que el polo del mediodía se elevaba fuera de nuestro horizonte cincuenta y dos grados y no veíamos las estrellas de la Osa Menor ni de la Mayor, estando alejados del puerto de donde partimos unas quinientas leguas por el siroco. Esto fue a tres días de abril" (61).

No olvidemos que la navegación se había hecho, siempre, "siguiendo el litoral" y "siguiendo esta playa". Así llegaron a los 52 grados de Latitud Sud. Era el 3 de abril de 1502.

"En este dia - dice Vespucci en la Lettera - se levantó en el mar una tormenta tan recia que nos hizo amainar del todo nuestras velas y corrimos a palo seco, con mucho viento, que era el lebeche, con olas grandísimas, y el aire tormentoso...".

Ahora sabemos que "por el siroco", el viente del sudeste, se habían alejado de la costa quinientas leguas. y que por "el lebeche", el viento del sudoeste, el 7 de abril, en medio de esa tormenta - en el paralelo 52 de Latitud Sud -, avistaron

"una nueva tierra, de la cual recorrimos cerca de veinte leguas, encontrando la costa brava, y no vimos en ella puerto alguno, ni gente, creo porque era el frío tan intenso que ninguno de la flota se podía remediar ni soportarlo" (62).

Así fueron descubiertas las islas que más tarde se llamaron Malvinas.

La cerrazón era tremenda; las olas, altísimas; las naves no podían verse las unas a las otras. El peligro no podía ser mayor. $\mathrm{El}$ capitán mayor hizo señales para que la flota se reuniese y retornase al camino de Portugal (63).

(61). - AMERICO VESPUCIO, El Mundo Nuevo cit. 259.

(62). - AMERICO VESPUCIO, EI Mundo Nuevo cit. 259.

(63). - AMERICO VESPUCIO, El Mundo Nuevo cit. 259. 
Así quedó abandonado, por tercera vez, el viaje al Oriente, al Sinus Magnus, a Ceilán y a las islas que debía haber más allá del Cabo Cattigara de Ptolomeo. Vespucci y su gente llegaron a Lisboa el 7 de septiembre de 1502 (64).

El descubrimiento de las Malvinas por Vespucci ha sido sostenido por algunos autores. El ingeniero Nicanor Alurralde lo ha defendido en una monografía que nosotros hemos apoyado y citado muchas veces. Las Malvinas están en el paralelo 52 de Latitud Sud. Vespucci no pudo ver otras tierras por la sencilla razón de que no existen y él no dice haber ido por otras partes. Pensar en las Georgias del Sud o en la Antártida es irrazonable. Y pensar en ballenas o témpanos es poco serio. La existencia de las Malvinas en el paralelo 52 y la coincidencia de haber llegado a ese paralelo Vespucci y haber dicho que allí vio una tierra no permite ninguna duda. La claridad de los hechos hace innecesario detenerse en algún error de imprenta o alguna confusión de cifras, rectificada por el propio Vespucci en otros escritos suyos.

La esperanza de alcanzar el Sinus Magnus, las islas tan decantadas por el aventurero Gaspar, hizo intervenir a Vespucci en un cuarto viaje. Cuenta Vespucci, en la Lettera, que

"partimos de este puerto de Lisboa seis naves en conserva con propósito de ir a descubrir una isla hacia el Oriente que se llama Malaccha, de la cual se tiene noticia que es muy rica" (65).

Agrega que Malaccha, o sea, Malaca,

"es como el almacén de todas las naves que vienen del mar Gangético y del mar Índico, como es Cádiz el lugar de reunión de todos los navios que pasan de levante a poniente".

Malaccha, repetimos, era como el almacén de todas las naves que venían del mar Gangético y del mar Indico

"y de poniente a levante por la vía de Calicut".

Hemos hecho esta repetición para leer bien este párrafo, algo confuso, pero preciso, que algunos historiadores han supuesto absurdo por creer que Vespucci consideraba a Cádiz un punto de reunión de

(64). - AMERICO VESPUCIO, El Mundo Nuevo cit. 261.

(65). - AMERICO VESPUCIO, El Mundo Nuevo cit. 261. 
todos los navíos que pasaban de levante a poniente y viceversa "por la vía de Calicut". En cambio, es un error colocar a Malaccha "más al occidente de Calicut", cuando está al oriente.

Otro error es decir que Calicut "está en un paraje a 33 grados del polo antártico". No es 33, sino sólo 3 . Error de imprenta, como otros (66).

Fue el cuarto y último fracaso de Vespucci. Por culpa del capitán mayor, unas naves se hundieron y las otras pudieron volver a Lisboa desde la costa del Brasil. Vespucci escribió su Lettera, con sus infortunios, desde esa ciudad, el 4 de septiembre de 1504 (67).

No olvidemos que en 1502, el mapa de Cantino mostraba la bandera portuguesa frente a Groenlandia con una inscripción en que recordaba que había sido descubierta por el rey don Manuel de Portugal y era "el final del Asia". Al noroeste de las Antillas del rey de España otra inscripción decía "Parte de Asia". El título del mapa expresaba que era el de las navegaciones de las islas descubiertas en las partes de la India. En el mapa de Contarini, de 1506, aparece la gran masa del Asia unida a América. En 1507, Martín Waldsemüller repetía el mapamundi de Ptolomeo y agregaba una isla con el nombre de América. Nadie dudaba que las tierras hoy llamadas América eran el Asia, la India Orientalis de Ptolomeo, entre el Atlántico y el Sinus Magnus, tal cual la había mostrado el mapamundi de Ptolomeo en el siglo II de nuestra Era y la había dibujado, grandiosa y enigmática, Enricus Martellus Germanus cuatro años antes del viaje de Colón. Por algo, el descubridor y Vespucci se habían empeñado tanto en hallar un paso que los condujese al otro mar, al Sinus Magnus, dibujado por Bartolomé Colón diez años antes del descubrimiento de Vasco Núñez de Balboa y perseguido, como una obsesión, en cuatro viajes fracasados, por ese poeta y navegante que se llamó Américo Vespucci.

ENRIQUE DE GANDIA, nacido en Buenos Aires en 1906. Doctor honoris causa por la Facultad de Derecho de la Asunción y por la Facultad de Filosofía y Letras de la Universidad Bartolomé Mitre de la Argentina. Miembro de número de la Academia Nacional de la Historia y de la de Ciencias Morales y Po-

(66). - AMERICO VESPUCIO, El Mundo Nuevo cit. 263.

(67). - AMERICO VESPUCIO, El Mundo Nuevo cit. 267. 
líticas (de la Argentina). Ex-rector, vicerector y decano. Profesor en tres Universidades. Dos premios nacionales de letras en la Argentina. Correspondiente de los Institutos Históricos y Geográficos de Río de Janeiro y San Pablo y de todas las Academias de Historia nacionales de América y las principales de España. Autor de cien libros de historia y mil quinientas monografías y artículos. Comendador de la Orden del Libertador de Venezuela. Medalla del reconocimiento paraguayo. Presidente de diversas instituciones culturales. Professor honorario y emérito de dos Universidades, etcétera.

Dirección: Calle Elflein, 3567

\section{LA LUCILA (Gran Buenos Aires)}

Argentina . 